\title{
The Analytic Solutions to the Task of Maintaining the Specified Characteristics Stability in Aircraft Flight Control System Reconfiguration
}

\author{
Dmitry Efanov ${ }^{1}$ and Vladislav Kosyanchuk ${ }^{2, *}$ \\ ${ }^{1}$ JSC "Spatial information systems", Moscow, Russia \\ ${ }^{2}$ FGUP "GosNIIAS", Moscow, Russia
}

\begin{abstract}
The paper sets out the conceptual issues of methods of analytical solution of the problem of defined aircraft stability characteristics conservation during flight control system reconfiguration under actuating subsystem failure. It is shown that the use of the reconfiguration technique with analytic set of stabilizing control laws synthesized by the decomposition method taking into account the cross-linking between the control channels allows to fully compensate the effects of failures of the actuating subsystem in each of the control channels under the conditions of complete controllability of aircraft as a dynamic system. It thus makes it possible to reconfigure the flight control system that always ensures the stability of the motion of the aircraft with a precisely prescribed poles. During study of failures in the form of a gradual reduction of the effectiveness of control surfaces it found that the synthesized analytical control law is a corresponding automatic increase feedback gain serviceable channel actuating subsystem, as well as the reallocation of control signals between the drives within the existing cross-connections between the control channels. Some of the solutions obtained by reconfiguring the control laws are not satisfactory, because of significantly limited possibility of maneuvering.
\end{abstract}

\section{Introduction}

Improving aircraft safety determines the relevance of the development of flight control systems capable of detecting failures and compensating for their effects. Modern aircraft are characterized by a high degree of analytical (functional) redundancy of actuating flight control system subsystems, so in the event of failure it is possible to redistribute functions.

At present, the functional (analytical) reconfiguration of the actuating subsystem has not been fully utilized.

There are passive and active approaches to reconfiguring the flight control system [1-2]. In the passive approach, the aim is to obtain a control law, which meets all the criteria set both in normal and emergency situations. An active approach is based on the failure identification scheme.

Development of analytical methods for reconfiguring the flight control system is a relatively new filed [3-4]. However, these studies do not cover sufficiently the task of ensuring the specified stability characteristics when reconfiguring the flight control system in case of failure and damage of the actuating subsystem elements. Therefore, the results obtained in these studies cannot be fully utilized to solve the reconfiguration problem.
The aim of this work is to find an analytical solution to maintain specified characteristics of the aircraft stability during flight control system reconfiguration.

\section{Results and discussions}

Consider a linear MIMO (Multi Input Multiple Output) system, the equations of which are defined as follows:

$$
\sigma \mathbf{x}(t)=\boldsymbol{A} \mathbf{x}(t)+\boldsymbol{B} \mathbf{u}(t), \mathbf{y}(t)=\boldsymbol{C} \mathbf{x}(t) .
$$

Where $t$ is time that can be either continuous $t \in \mathbb{R}_{+}=[0,+\infty)$, or discrete $t \in \mathbb{N}_{0}=\{0,1,2, \ldots\}$. The vector $\mathbf{x} \in \mathbb{R}^{n}$ corresponds to the dimension state vector $n ; \mathbf{u} \in \mathbb{R}^{r}$ to the dimension input (control) vector $r$; $\mathbf{y} \in \mathbb{R}^{m}$ to the output (measurement) vector $m$. Operator $\sigma$ means either time differentiation for the continuous system, i.e. $\sigma \mathbf{x}(t)=\dot{\mathbf{x}}(t)$, or time offset operation for the discrete system, i.e. $\sigma \mathbf{x}(t)=\mathbf{x}(t+1)$. The set $\mathbb{R}$ means real numbers (real axis), and $\mathbb{R}^{k}$ means the dimension real space $k$ accordingly.

It is believed that the set of eigenvalues of matrix $\boldsymbol{A}$

$$
\operatorname{eig}(\boldsymbol{A})=\left\{\lambda_{i} \in \mathbb{C}: \operatorname{det}\left(\lambda \boldsymbol{I}_{n}-\boldsymbol{A}\right)=0\right\}
$$

inevitably includes such complex numbers $\lambda_{i} \in \mathbb{C}$ that their real parts $\operatorname{Re}\left(\lambda_{i}\right)>0$ for the case $\sigma \mathbf{x}(t)=\dot{\mathbf{x}}(t)$, or

Corresponding author: kos.vl.v@gmail.com 
their modulus $\left|\lambda_{i}\right|>1$ for the case $\sigma \mathbf{x}(t)=\mathbf{x}(t+1)$. Here $\boldsymbol{I}_{n}$ is an identity matrix of order $n ;\left|\lambda_{i}\right|$ is an eigenvalue modulus $\lambda_{i} ; \mathbb{C}$ is a set of complex numbers (complex plane).

We introduce the concept of set $\mathbb{C}^{\text {stab }}$, which represents, depending on the type of the dynamic system (continuous or discrete one) respectively left halfplane $\mathbb{C}^{-}$, i.e. $\mathbb{C}^{\text {stab }} \doteq \mathbb{C}^{-}$, or the area within a circle of unit radius centered at the beginning of $\mathbb{C}$, i.e. $\mathbb{C}^{\text {stab }} \doteq \mathbb{C}_{|\lambda|<1}$. Here $\operatorname{eig}(\boldsymbol{A}) \subset \mathbb{C}^{\text {stab }}$ is a condition of asymptotic stability expressed in terms of eigenvalues (poles) of the matrix.

It is assumed that for the MIMO-system (1) some control exists with a negative feedback of type:

$$
\mathbf{u}(t)=-\boldsymbol{K} \mathbf{x}(t),
$$

where $\boldsymbol{K} \in \mathbb{R}^{r \times n}$ is a matrix of coefficients (regulator matrix).

Assume that $\boldsymbol{B}^{\perp}$ is a zero divisor of the matrix meeting the following conditions: $\boldsymbol{B}^{\perp} \boldsymbol{B}=\mathbf{0}_{(n-r) \times r}$, $\boldsymbol{B}^{\perp} \boldsymbol{B}^{\perp \mathrm{T}}=\boldsymbol{I}_{n-r}$, where $\boldsymbol{B}^{+}$is the Moore-Penrose pseudoinverse; $\mathbf{0}_{r \times r}$ is a zero matrix of size $r \times r$.

Consider the following multi-level decomposition of the dynamical system (1) with matrices $\boldsymbol{A} \in \mathbb{R}^{n \times n}, \boldsymbol{B} \in \mathbb{R}^{n \times m}$ and $\boldsymbol{C}=\boldsymbol{I}_{n}[5]$.

The zero (original) level is

$$
\boldsymbol{A}_{0}=\boldsymbol{A}, \boldsymbol{B}_{0}=\boldsymbol{B},
$$

the first level is

$$
\boldsymbol{A}_{1}=\boldsymbol{B}^{\perp} \boldsymbol{A} \boldsymbol{B}^{\perp \mathrm{T}}, \boldsymbol{B}_{1}=\boldsymbol{B}^{\perp} \boldsymbol{A B},
$$

$k$ th (intermediate) level is

$$
\boldsymbol{A}_{k}=\boldsymbol{B}_{k-1}^{\perp} \boldsymbol{A}_{k-1} \boldsymbol{B}_{k-1}^{\perp \mathrm{T}}, \boldsymbol{B}_{k}=\boldsymbol{B}_{k-1}^{\perp} \boldsymbol{A}_{k-1} \boldsymbol{B}_{k-1},
$$

Lth (final) level is $L=\operatorname{ceil}(n / r)-1$,

$$
\boldsymbol{A}_{L}=\boldsymbol{B}_{L-1}^{\perp} \boldsymbol{A}_{L-1} B_{L-1}^{\perp \mathrm{T}}, B_{L}=B_{L-1}^{\perp} \boldsymbol{A}_{L-1} \boldsymbol{B}_{L-1} .
$$

Here ceil $(*)$ is rounding operation for numbers $*$ to the greater value, e.g., $\quad \operatorname{ceil}(0.3)=1, \quad \operatorname{ceil}(1.1)=2$, $\operatorname{ceil}(2.01)=3$, etc.

If the MIMO system (1) with two matrices $(\boldsymbol{A}, \boldsymbol{B})$ is fully controllable, then all systems with matrices $\left(\boldsymbol{A}_{i}, \boldsymbol{B}_{i}\right)(4)-(5)$ are controllable.

Assume that the MIMO system (1) is fully controllable and all matrices $\boldsymbol{B}_{i}$ have full ranks in rows, and matrix $\boldsymbol{K} \in \mathbb{R}^{r \times n}$ meets "assembly" formulas:

$$
\begin{gathered}
\boldsymbol{K}_{=} \boldsymbol{K}_{0}=\boldsymbol{B}_{0}^{-} \boldsymbol{A}-\Phi_{0} \boldsymbol{B}_{0}^{-}, \quad \boldsymbol{B}_{0}^{-}=\boldsymbol{B}_{0}^{+}+\boldsymbol{K}_{1} \boldsymbol{B}_{0}^{\perp}, \\
\boldsymbol{K}_{1}=\boldsymbol{B}_{1}^{-} \boldsymbol{A}_{1}-\Phi_{1} \boldsymbol{B}_{1}^{-}, \quad \boldsymbol{B}_{1}^{-}=\boldsymbol{B}_{1}^{+}+\boldsymbol{K}_{2} \boldsymbol{B}_{1}^{\perp}, \ldots \\
\boldsymbol{K}_{k}=\boldsymbol{B}_{k}^{-} \boldsymbol{A}_{k}-\Phi_{k} \boldsymbol{B}_{k}^{-}, \quad \boldsymbol{B}_{k}^{-}=\boldsymbol{B}_{k}^{+}+\boldsymbol{K}_{k+1} \boldsymbol{B}_{k}^{\perp}, \ldots \\
\boldsymbol{K}_{L}=\boldsymbol{B}_{L}^{-} \boldsymbol{A}_{L}-\Phi_{L} \boldsymbol{B}_{L}^{-},
\end{gathered}
$$

Then the following equation is correct:

$$
\operatorname{eig}(\boldsymbol{A}-\boldsymbol{B} \boldsymbol{K})=\bigcup_{i=1}^{L+1} \operatorname{eig}\left(\Phi_{i-1}\right) .
$$

The limitation of rank completeness for matrices $\boldsymbol{B}_{i}$ is in contrast with the presence of functional redundancy of the flight control system; therefore an algorithm has been designed to eliminate this limitation. It comprises the following steps:

1) check the rank of matrix $\boldsymbol{B}$;

2) if $\operatorname{rank} \boldsymbol{B}<r$, then perform a skeleton decomposition $\boldsymbol{B}=\widehat{\boldsymbol{B}} \boldsymbol{T}$;

3) define matrices $\boldsymbol{A}_{0}=\boldsymbol{A}, \boldsymbol{B}_{0}=\widehat{\boldsymbol{B}}$;

4) calculate $L=\operatorname{ceil}(n / r)-1$;

5) set matrices $\Phi=\Phi_{0}, \Phi_{1}, \ldots, \Phi_{L}$, such that $\bigcup_{i=1}^{L+1} \operatorname{eig}\left(\Phi_{i-1}\right)$ a desired set of eigenvalues of the closed system;

6) calculate the zero divisor $\boldsymbol{B}_{0}^{\perp}$, and then matrices $\boldsymbol{A}_{1}=\boldsymbol{B}_{0}^{\perp} \boldsymbol{A}_{0} \boldsymbol{B}_{0}^{\perp+}, \quad \boldsymbol{B}_{1}=\boldsymbol{B}_{0}^{\perp} \boldsymbol{A}_{0} \boldsymbol{B}_{0}, \ldots, \quad \boldsymbol{A}_{k}=\boldsymbol{B}_{k-1}^{\perp} \boldsymbol{A}_{k-1} \boldsymbol{B}_{k-1}^{\perp+}$, $\boldsymbol{B}_{k}=\boldsymbol{B}_{k-1}^{\perp} \boldsymbol{A}_{k-1} \boldsymbol{B}_{k-1}$;

7) calculate the zero divisor $\boldsymbol{B}_{k}^{\perp}$, and then matrices $\boldsymbol{A}_{k+1}=\boldsymbol{B}_{k}^{\perp} \boldsymbol{A}_{k} \boldsymbol{B}_{k}^{\perp+}, \boldsymbol{B}_{k+1}=\boldsymbol{B}_{k}^{\perp} \boldsymbol{A}_{k} \boldsymbol{B}_{k}, \ldots$;

8) determine the zero divisor $\boldsymbol{B}_{L-2}^{\perp}$, and then matrices $\boldsymbol{A}_{L-1}=\boldsymbol{B}_{L-2}^{\perp} \boldsymbol{A}_{L-2} \boldsymbol{B}_{L-2}^{\perp+}, \boldsymbol{B}_{L-1}=\boldsymbol{B}_{L-2}^{\perp} \boldsymbol{A}_{L-2} \boldsymbol{B}_{L-2} ;$

9) calculate the zero divisor $\boldsymbol{B}_{L-1}^{\perp}$, and then matrices $\boldsymbol{A}_{L}=\boldsymbol{B}_{L-1}^{\perp} \boldsymbol{A}_{L-1} \boldsymbol{B}_{L-1}^{\perp+}, \boldsymbol{B}_{L}=\boldsymbol{B}_{L-1}^{\perp} \boldsymbol{A}_{L-1} \boldsymbol{B}_{L-1} ;$

10) successively calculate matrices

$\boldsymbol{K}_{L}=\boldsymbol{B}_{L}^{-} \boldsymbol{A}_{L}-\Phi_{L} \boldsymbol{B}_{L}^{-}$,

$\boldsymbol{B}_{L-1}^{-}=\boldsymbol{K}_{L} \boldsymbol{B}_{L-1}^{\perp}+\boldsymbol{B}_{L-1}^{+}, \boldsymbol{K}_{L-1}=\boldsymbol{B}_{L-1}^{-} \boldsymbol{A}_{L-1}-\Phi_{L-1} \boldsymbol{B}_{L-1}^{-}, \ldots$,

$\boldsymbol{B}_{k}^{-}=\boldsymbol{K}_{k+1} \boldsymbol{B}_{k}^{\perp}+\boldsymbol{B}_{k}^{+}, \boldsymbol{K}_{k}=\boldsymbol{B}_{k}^{-} \boldsymbol{A}_{k}-\Phi_{k} \boldsymbol{B}_{k}^{-}, \ldots$,

$\boldsymbol{B}_{1}^{-}=\boldsymbol{K}_{2} \boldsymbol{B}_{1}^{\perp}+\boldsymbol{B}_{1}^{+}, \boldsymbol{K}_{1}=\boldsymbol{B}_{1}^{-} \boldsymbol{A}_{1}-\Phi_{1} \boldsymbol{B}_{1}^{-}$,

$\boldsymbol{B}_{0}^{-}=\boldsymbol{K}_{1} \boldsymbol{B}_{0}^{\perp}+\boldsymbol{B}_{0}^{+}, \boldsymbol{K}=\boldsymbol{T}^{+} \boldsymbol{K}_{0}=\boldsymbol{T}^{+}\left(\boldsymbol{B}_{0}^{-} \boldsymbol{A}_{0}-\Phi_{0} \boldsymbol{B}_{0}^{-}\right)$.

The obtained regulator

$$
\boldsymbol{K}=\boldsymbol{T}^{+} \boldsymbol{K}_{0}=\boldsymbol{T}^{+}\left(\boldsymbol{B}_{0}^{-} \boldsymbol{A}_{0}-\Phi_{0} \boldsymbol{B}_{0}^{-}\right), \boldsymbol{B}_{0}^{-}=\boldsymbol{K}_{1} \boldsymbol{B}_{0}^{\perp}+\boldsymbol{B}_{0}^{+}
$$

ensures fulfilment of condition (11), that is the condition of specified pole placement.

Based on the analysis of the relationships in the discussed decomposition method of pole placement, sources of equivalent control laws are identified. There are three sources of equivalent solutions in the form of control laws, i.e. a set of $\boldsymbol{K}_{\hat{\}}}$ regulator matrices, for which the following equation is satisfied

$$
\operatorname{eig}\left(\boldsymbol{A}-\boldsymbol{B} \boldsymbol{K}_{\}}\right)=\bigcup_{i=1}^{L+1} \operatorname{eig}\left(\Phi_{i-1}\right) \text {. }
$$

1. From the guidelines of the above method and the pole placement algorithm constructed on its basis, it follows that, in general, no restriction is imposed on matrices $\Phi_{i-1}$ except the conformity of their sets of values with the predetermined values.

2. Another source for a set of equivalent laws $\boldsymbol{K}_{\hat{\}}}$ is the loss of the full rank in the columns of matrices $\boldsymbol{B}_{i}$ in the decomposition process (3) - (6).

Consider the "skeleton" decomposition $\boldsymbol{B}_{i}=\widehat{\boldsymbol{B}}_{i} \boldsymbol{T}_{i}$ in case of loss of the full rank in the columns of matrix $\boldsymbol{B}_{i}$. In this case, the equation of sets of eigenvalues is satisfied 


$$
\operatorname{eig}\left(\boldsymbol{A}_{i}-\widehat{\boldsymbol{B}}_{i} \widehat{\boldsymbol{K}}_{i}\right)=\operatorname{eig}\left(\boldsymbol{A}_{i}-\boldsymbol{B}_{i} \boldsymbol{T}_{i}^{+} \widehat{\boldsymbol{K}}_{i}\right),
$$

where $\boldsymbol{T}_{i} \boldsymbol{T}_{i}^{+}=\boldsymbol{I}$. If, according to formula (12) matrix $\boldsymbol{T}_{i}^{+} \widehat{\boldsymbol{K}}_{i}$ is completed with a summand of the zero divisor, $\boldsymbol{B}_{i R}^{\perp} \varpi_{i}$, where $\boldsymbol{B}_{i R}^{\perp}$ is the right zero divisor of matrix $\boldsymbol{B}_{i}$, i.e. $\boldsymbol{B}_{i} \boldsymbol{B}_{i R}^{\perp}=0, \varpi_{i}$ is a matrix of appropriate size with arbitrary elements, then the following equation will be satisfied

$$
\operatorname{eig}\left(\boldsymbol{A}_{i}-\hat{\boldsymbol{B}}_{i} \hat{\boldsymbol{K}}_{i}\right)=\operatorname{eig}\left(\boldsymbol{A}_{i}-\boldsymbol{B}_{i}\left(\boldsymbol{T}_{i}^{+} \hat{\boldsymbol{K}}_{i}+\boldsymbol{B}_{i R}^{\perp} \varpi\right)\right) .(14
$$

Thus, the addition to regulators $\widehat{\boldsymbol{K}}_{i}$ obtained at each level of decomposition of the mathematical model of the MIMO system (1) generates a proper subset of the set of equivalent control laws.

3. Finally, the third source generating the equivalent laws is an incomplete rank in the columns of matrix $\boldsymbol{B}=\boldsymbol{B}_{0}$ of the model (1). This source from a formal point of view falls under the above discussions about the "skeleton" decomposition of $\boldsymbol{B}_{i}=\widehat{\boldsymbol{B}}_{i} \boldsymbol{T}_{i}$. However, it is placed as a separate category because only addition $\boldsymbol{B}_{0 R}^{\perp} \varpi_{i}=\boldsymbol{B}_{R}^{\perp} \varpi_{i} \quad$ (at the zero level of decomposition) is additive and all the others can be regarded as multiplicative due to the "assembly" procedures of the regulator (7) - (10).

The mid-range passenger plane considered in the paper is made by the normal scheme. The arrow wing is low and high-lift (slats and single-slotted flaps). The vertical tail is single-fin; the horizontal stabilizer is located on the rear fuselage. Aerodynamic pitch is controlled by a stabilizer (balancing) and elevators, roll is controlled by ailerons, and yaw is controlled by a rudder.

To be definite in solving problems of reconfiguring the flight control system, we will use a continuous linear model of aircraft motion, i.e. in this case $\sigma \mathbf{x}(t)=\dot{\mathbf{x}}(t)$. The state vector of the aircraft model (1) is as follows

$$
\mathbf{x}=\left(\begin{array}{llllllll}
V & \alpha & \Theta & \omega_{z} & \beta & \omega_{x} & \omega_{y} & \gamma
\end{array}\right)^{\mathrm{T}} \in \mathbb{R}^{8},
$$

where $\omega_{x}, \omega_{y}, \omega_{z}$ are angular velocities of roll, yaw and pitch, respectively; $\alpha, \Theta, \gamma, \beta$ are angles of attack, flight path, roll and glide, respectively; $V$ is flight velocity. The aircraft model control vector (1) is

$$
\mathbf{u}=\left(\begin{array}{lllll}
\delta_{\text {elevator }}^{\text {left }} & \delta_{\text {elevator }}^{\text {right }} \quad \delta_{\text {rudder }} & \delta_{\text {aileron }}^{\text {left }} & \delta_{\text {aileron }}^{\text {right }}
\end{array}\right)^{\mathrm{T}} \in \mathbb{R}^{5},
$$

where $\delta_{\text {elevator }}^{\text {left }}, \delta_{\text {elevator }}^{\text {righ }}$ are deflection angles of the left and right elevators, respectively, with deflection ranges of ($\left.27^{\circ} \ldots 22^{\circ}\right) ; \delta_{\text {rudder }}$ is rudder deflection angle $\left(-27^{\circ} \ldots 27^{\circ}\right)$; $\delta_{\text {aileron }}^{\text {left }}, \delta_{\text {aileron }}^{\text {right }}$ angles of the left and right ailerons, respectively, with deflection ranges of $\left(-27^{\circ} \ldots 15^{\circ}\right)$.

Using the methodology of dividing the motion into disturbance and non-disturbance motion, the matrix representation of longitudinal and lateral motion of the aircraft has the following form

$$
\begin{gathered}
\boldsymbol{A}=\left(\begin{array}{cccc:cccc}
a_{11} & a_{12} & a_{13} & 0 & 0 & 0 & 0 & 0 \\
a_{21} & a_{22} & a_{23} & 1 & 0 & 0 & 0 & 0 \\
-a_{21} & -a_{22} & -a_{23} & 0 & 0 & 0 & 0 & 0 \\
a_{41} & a_{42} & a_{43} & a_{44} & 0 & 0 & 0 & 0 \\
\hdashline 0 & 0 & 0 & 0 & a_{55} & a_{56} & a_{57} & a_{58} \\
0 & 0 & 0 & 0 & a_{65} & a_{66} & a_{67} & 0 \\
0 & 0 & 0 & 0 & a_{75} & a_{76} & a_{77} & 0 \\
0 & 0 & 0 & 0 & 0 & 1 & a_{87} & 0
\end{array}\right), \\
\boldsymbol{B}=\left(\begin{array}{cc:cccc}
0 & 0 & 0 & 0 & 0 \\
b_{21} & b_{22} & 0 & b_{25} & b_{26} \\
-b_{21} & -b_{22} & 0 & -b_{25} & -b_{26} \\
b_{41} & b_{42} & 0 & b_{45} & b_{46} \\
0 & 0 & b_{54} & 0 & 0 \\
b_{61} & -b_{62} & b_{64} & b_{65} & -b_{66} \\
b_{71} & -b_{72} & b_{74} & b_{75} & -b_{76} \\
0 & 0 & 0 & 0 & 0
\end{array}\right) . \quad(15)
\end{gathered}
$$

As can be seen from the structure of the control efficiency matrix $\boldsymbol{B}$, this aircraft model allows for cross couplings between control channels (controls) of the aircraft for longitudinal and lateral motion.

Since the change of the aircraft flight mode effects aerodynamic coefficients $a_{i j}$ and $b_{i j}$, it is assumed that failures and damage of the actuating subsystem of the aircraft flight control system also lead to changes in coefficients $b_{i j}$, and in case of full loss of control they go down to zero. The worst cases of failures in the channel of the elevator and ailerons of the actuating subsystem are associated with a complete violation of the mutual "symmetry" of coefficients in the matrix $\boldsymbol{B}$, with which inequalities $b_{i 1} \neq b_{i 2}, \quad b_{i 5} \neq b_{i 6}, i \in\{2,4,6,7\}$ are satisfied.

In this paper, the problem of reconfiguring the flight control system was solved analytically, i.e., without numerical errors. To this end, two approaches to solving this problem were considered.

1. Sources are analyzed that generate a set of equivalent control laws $\boldsymbol{K}_{\{\}}$, namely, 1) freedom to choose (set) matrices $\Phi_{i-1}$ in (7) - (10); 2) incomplete rank in columns of matrix $\boldsymbol{B}_{i}$ in the decomposition procedure $(3)-(6)$; 3) incomplete rank in columns of matrix $\boldsymbol{B}=\boldsymbol{B}_{0}$ in the initial model of the MIMO system (1). In the latter case, the control law includes explicitly an additive component of $\boldsymbol{B}_{R}^{\perp} \varpi$.

To obtain calculated ratios of analytical reconfiguration of the flight control system, it is believed that an operating object and an object with a failure of the actuating subsystem are presented by corresponding linear MIMO-systems of type (1):

$$
\begin{gathered}
\sigma \mathbf{x}(t)=\boldsymbol{A} \mathbf{x}(t)+\boldsymbol{B} \mathbf{u}(t), \mathbf{y}(t)=\mathbf{x}(t), \boldsymbol{C}=\boldsymbol{I}_{n}, \\
\sigma \mathbf{x}_{\text {failure }}(t)=\boldsymbol{A} \mathbf{x}_{\text {failure }}(t)+\boldsymbol{B}_{\text {failure }} \mathbf{u}_{\text {failure }}(t), \\
\mathbf{y}_{\text {failure }}(t)=\mathbf{x}_{\text {failure }}(t), \boldsymbol{C}_{\text {failure }}=\boldsymbol{I}_{n} .
\end{gathered}
$$


Thus, the failure of the actuating subsystem of the flight control system is modeled by matrix $\boldsymbol{B}_{\text {failure }}$. Here all variables of the operating and failed objects are observed. It is also believed that for matrix $\boldsymbol{B}$ the rank of the columns can be equal to or less than the number of columns $r$, which in turn is no less than the dimension of the state space, i.e. $\operatorname{rank} \boldsymbol{B} \leq r<n$.

Control laws for the properly operating aircraft and the flight control system of the reconfigured object correspond to the following equations, respectively, $\mathbf{u}(t)=-\boldsymbol{K} \mathbf{x}(t), \mathbf{u}_{\text {failure }}(t)=-\boldsymbol{K}_{\text {rec }} \mathbf{x}_{\text {failure }}(t)$, where $\boldsymbol{K}_{\text {rec }}$ is a matrix of the reconfigured control law.

It is believed that the regulator matrix in the control law for the properly operating flight control system is given by (7)

$$
\boldsymbol{K}=\boldsymbol{B}_{0}^{-} \boldsymbol{A}-\Phi \boldsymbol{B}_{0}^{-}+\boldsymbol{B}_{R}^{\perp} \varpi, \quad \boldsymbol{B}_{0}^{-}=\boldsymbol{B}_{0}^{+}+\boldsymbol{K}_{1} \boldsymbol{B}_{0}^{\perp},
$$

and the matrix of reconfigured regulator is $\boldsymbol{K}_{\text {rec }} \in \boldsymbol{K}_{\}}$. Then the problem of reconfiguration will be to ensure equality of eigenvalues sets:

$$
\operatorname{eig}\left(\boldsymbol{A}-\boldsymbol{B}_{\text {failure }} \boldsymbol{K}_{\{\cdot\}}\right)=\operatorname{eig}(\boldsymbol{A}-\boldsymbol{B} \boldsymbol{K}),
$$

where $\boldsymbol{K}_{\text {rec }}=\boldsymbol{K}_{\{\cdot\}}$ is an appropriate element of the analytic set of control laws $\boldsymbol{K}_{\{}$providing the desired placement of the poles. Here the class condition (belonging to the considered regulator set of the properly operating flight control system) is also met, i.e.

$$
\left.\begin{array}{c}
\boldsymbol{K}=\boldsymbol{B}_{0}^{-} \boldsymbol{A}-\Phi \boldsymbol{B}_{0}^{-}+\boldsymbol{B}_{R}^{\perp} \varpi \\
\boldsymbol{B}_{0}^{-}=\boldsymbol{B}_{0}^{+}+\boldsymbol{K}_{1} \boldsymbol{B}_{0}^{\perp}
\end{array}\right\} \in \boldsymbol{K}_{\}} .
$$

2. Another approach to reconfiguring the flight control system is an analytical synthesis of a set of control laws $\boldsymbol{K}_{0\}}$ for the "worst" (in some sense) case of failure of the actuating subsystem. The control law under normal functioning of the flight control system, as well as all others failures less dangerous for flight safety will be elements of this set of control laws.

\section{Conclusions}

Thus, the main provisions of the analytical solution are formulated for the problem of maintaining the specified aircraft stability characteristics in case of the flight control system reconfiguration due to failures and malfunctions of the actuating subsystem. The singularity of these results is that implementation and adaptation of the developed algorithms in on-board control complexes of modern and advanced aircraft will ensure a high level of flying qualities for states of failure to provide safe continuation and completion of the flight.

It is shown that the decomposition method of pole placement used in the synthesis of stabilizing control laws can be applied to reconfigure the flight control system. The analytical synthesis of control laws was demonstrated, as well as the procedure for constructing a set of equivalent solutions on the basis of identified sources of ambiguity (freedom of choice).

\section{References}

1. T. A. Johansen, T. I. Fossen, Automatica, 49(5), 1087-1103 (2013)

2. X. Yu, J. Jiang, Annual Reviews in Control, 39, 46$57(2015)$

3. E. Yu. Zybin, V. V. Kosyanchuk, Journal of Computer and Systems Sciences International, 49(1), 105-114 (2010)

4. E. Yu. Zybin, V. V. Kosyanchuk, A. M. Kulchak, Mekhatronika, Avtomatizatsiya, Upravlenie, 7, 5966 (2014)

5. E.A. Mikrin, V.N. Ryabchenko, N.E. Zubov, Matrix methods in the theory and practice of automatic control systems of aircraft (Moscow, Publishing House of N.E. Bauman, 2016) 